\title{
Variability in Surgical Treatment of Spondylolisthesis among Spine Surgeons
}

Daniel Lubelski, MD ${ }^{1,5}$, Vincent J Alentado, MD ${ }^{1,6}$, Seth K. Williams, MD², Colin

O’Rourke, MS ${ }^{1}$, Nancy A. Obuchowski, PhD ${ }^{1}$, Jeffrey C. Wang, MD³, Michael P.

Steinmetz, MD¹, Alfred J. Melillo, PA${ }^{1}$, Edward C Benzel, MD¹, Michael T. Modic, MD¹, Robert Quencer, MD4, Thomas E. Mroz, MD¹

1 Cleveland Clinic, Cleveland, $\mathrm{OH}$

2 University of Wisconsin Department of Orthopedics and Rehabilitation

3 University of Southern California, Los Angeles, CA

${ }^{4}$ University of Miami Miller School of Medicine, Miami, FL

5 Johns Hopkins Hospital, Baltimore, MD

6 Indiana University, Indianapolis, IN

Corresponding Author:

Thomas E. Mroz, MD

Cleveland Clinic Foundation

9500 Euclid Avenue, S40

Cleveland, Ohio 44195

Ph: 216-445-9232

Fax: 216-445-6801

mrozt@ccf.org

This is the author's manuscript of the article published in final edited form as:

Lubelski, D., Alentado, V. J., Williams, S. K., O’Rourke, C., Obuchowski, N. A., Wang, J. C., ... Mroz, T. E. (2018). Variability in Surgical Treatment of Spondylolisthesis Among Spine Surgeons. World Neurosurgery, 111, e564-e572. https://doi.org/10.1016/j.wneu.2017.12.108 


\section{Abstract}

Background context: There are a multitude of surgical and nonsurgical treatments for low grade lumbar spondylolisthesis. Treatments include non-operative, decompression without fusion, and instrumented fusion. There are no clear guidelines for the optimal approach.

Purpose: To identify the surgical treatment patterns for spondylolisthesis, among United States spine surgeons

Patient Sample: 445 orthopedic and neurosurgery US spine surgeons were surveyed

Study design: Electronic survey

Outcome measures: 1) Differences in surgical treatment responses based on various demographic variables; 2) probability of disagreement based on surgeon subgroups

Methods: 445 US spine surgeons completed a survey of clinical and radiographic case scenarios on patients with lumbar spondylolisthesis with stenosis and neurogenic claudication with (S1) or without (S2) associated mechanical back pain. Surgical treatment options included laminectomy/foraminotomy, laminectomy and in-situ fusion, laminectomy with posterolateral fusion using pedicle screws (PLF), posterior lumbar interbody fusion (PLIF/TLIF), or none of the above. Statistical significance was set at 0.01 to account for multiple comparisons.

Results: There was 64\% disagreement among surgeons for treatment of spondylolisthesis with mechanical back pain (S1) and 71\% disagreement for spondylolisthesis without mechanical back pain (S2). For S1, disagreement was $52 \%$ for those practicing 5-10 years versus 70\% among those practicing $>20$ years. Orthopedic surgeons had greater disagreement compared to neurosurgeons (76\% vs $56 \%$ ) for S1. Greater clinical equipoise was seen for S2 compared to S1 regardless of surgeon characteristics. For spondylolisthesis without mechanical back pain, neurosurgeons were significantly more likely to select decompression-only as compared to orthopedic surgeons who more commonly fused. Other differences in operative decisions were seen based on duration in practice and case volume.

Conclusions: Substantial clinical equipoise exists among US spine surgeons for the treatment of spondylolisthesis with or without low back pain. Differences in operative practice are higher when the patient presents without associated back pain. Surgeon case volume, practice duration, and specialty training influence operative decisions for a given pathology. Recognizing this practice variation will 
hopefully lead to better evidence and practice guidelines for the optimal and most cost effective treatment paradigms.

\section{Introduction}

Degenerative spondylolisthesis (DS) is a common cause of spinal stenosis and leads to low back pain, radiculopathy, and neurogenic claudication. Surgery is the mainstay treatment in patients that are refractory to conservative management and in those with weakness or neurological symptoms. ${ }^{1}$

The Spine Patient Outcomes Research Trial (SPORT) ${ }^{2}$ found that among patients with degenerative spondylolisthesis and spinal stenosis that are treated surgically pain and function are superior over a period of two years as compared to those treated nonsurgically. Moreover, a post-hoc analysis ${ }^{3}$ suggested that patients with predominant leg pain improved significantly more than those with predominant back pain. Despite numerous studies, the optimal surgical treatment paradigm is still unclear. Early studies reported the use of decompression without fusion for DS, with $69-82 \%$ showing satisfactory outcomes. ${ }^{4-7}$ A randomized trial by Herkowitz and Kurz ${ }^{8}$ showed that decompression and non-instrumented fusion had superior back and leg pain outcomes compared to those that underwent laminectomy alone. In recent years, instrumented fusion has become increasingly popular following studies showing improved fusion rates. ${ }^{9}$ The type of fusion is also in question. 2009 North American Spine Society guidelines stated that there was insufficient evidence to make a recommendation regarding interbody fusion versus posterolateral fusion. ${ }^{1} \mathrm{~A}$ recent retrospective study by $\mathrm{Ha}$ et $\mathrm{al},{ }^{10}$ however, found that while interbody versus posterolateral fusion was not significantly different among stable DS patients, among those with unstable DS, addition of an interbody provided superior postoperative pain relief.

Ultimately, there is substantial clinical equipoise among spine surgeons regarding the optimal surgical approach for patients with degenerative spondylolisthesis. The treatment paradigm is further complicated depending on whether the presenting 
symptoms are mainly mechanical back pain versus predominantly leg pain. The variability in approaches and indications for surgery is related to the limited high quality evidence. Evolving surgical treatments, development of novel minimally invasive approaches, and increasing emphasis on cost effectiveness further complicates the decision tree. Financial incentives, differences in specialty and training backgrounds, as well as variability in practice cultures based on geographical region and practice type also influences surgical approach.

In an era of value-based health care, there is increased scrutiny regarding surgical decision-making and resource utilization. With approximately 3000 U.S. spine surgeons performing more than 400,000 spine fusions annually, ${ }^{11}$ at a high cost to the health care system, understanding of optimal and most cost effective surgical treatment paradigms for given pathologies is imperative.

The present study includes a subset of a larger survey ${ }^{11}$ that evaluated surgical treatment patterns among spine surgeons nationally for several common spinal pathologies. In the present study, we report the results pertaining to the treatment of spondylolisthesis with or without associated mechanical back pain. We hypothesized that there are substantial differences in the types of surgeries selected for the treatment of spondylolisthesis. As for other pathologies, we anticipated that this lack of uniformity would be influenced by surgeon's geographical location, specialty training, years of experience, and the practice type.

\section{Materials and Methods}

An online survey was developed, using the RedCAP Database ${ }^{12}$ (Research Electronic Data Capture, Nashville, TN), to assess surgeon practice patterns for common lumbar pathologies. Details of this national survey have been previously published. ${ }^{11}$ In short, those surveyed were asked to provide details regarding the geographical location of their practice, their specialty, fellowship training, type of 
practice (Private, Academic, Hybrid), number of spine surgeries performed per year, years in practice, use of discograms, and whether or not the surgeon typically surgically treats back pain due to degenerative disc disease. Subsequently, the survey participants were asked clinical questions and provided with complementary XR and magnetic resonance images.

To assess practice patterns among surgeons for the treatment of spondylolisthesis, the following two case scenarios were constructed: Scenario 1 (S1) 51 year old male with L4-5 Spondylolisthesis with stenosis and correlative neurogenic claudication WITH mechanical back pain; and Scenario 2 (S2) 51 year old male with L4-5 Spondylolisthesis with stenosis and correlative neurogenic claudication WITHOUT mechanical back pain (Figures 1-2). The patients in each scenario had failed nonoperative care. The answer choices for treatment options in both scenarios were laminectomy/foraminotomy, laminectomy and in-situ fusion, laminectomy with posterolateral fusion using pedicle screws (PLF), posterior/transformainal lumbar interbody fusion (PLIF/TLIF), anterior lumbar interbody fusion (ALIF) with percutaneous pedicle screws, ALIF with open posterior instrumentation, interspinous spacer device, lateral interbody fusion (LIF; eg DLIF, XLIF), or none of the above.

The survey was electronically sent to 2460 spine surgeons selected randomly from a national spine surgeon database. The respondents' names were blinded, and the results of completed surveys were analyzed using R software (version 2.15.0, Vienna, Austria) and SAS software (version 9.3, Cary, NC). The data were summarized in two-way contingency tables using counts and percentages. Pearson's chi-squared statistic was used to test significance of the relationships between demographic and surgeon response variables. Statistical significance was set at 0.01 to account for multiple comparisons. Multinomial logistic regression models and odds ratios comparing the relative frequencies between physician groups were used to characterize dependencies across physician groups. 
We also calculated the overall probability of two randomly chosen surgeons disagreeing on the treatment method, given a particular scenario. Treatment variability found within specific subsets of surgeons was compared to the overall level of treatment variability found within all surveyed surgeons. Nonparametric bootstrap methods were used to create $95 \%$ confidence intervals for the true odds ratios comparing disagreement between surgeons. Ten thousand bootstrap replicates were used to create each confidence interval, which were based on percentiles of the bootstrap distribution of the statistic.

\section{Results}

Four hundred and forty-five of 2460 (18\%) surgeons completed the survey. The demographic results of the respondents are summarized in Table 1. The Midwest represented 28\% of respondents, North East was 24\%, South East was 22\%, and while the West and South West were 15\% and 12\%, respectively. Of the 445 participants, 318 (75\%) were orthopaedic surgeons and 107 (25\%) were neurological surgeons. Eighty percent (340 of 445 respondents) were fellowship trained. Fifty-seven percent (241 of 445) were private practitioners, 20\% (85 of 445) reported Hybrid practices (i.e. a combination of academic and private practice), and 23\% (99 of 445) were academicians. There was a wide range of reported case volumes with the most common (96 of 445, 23\%) being between 151200 cases per year. Similarly, there was a wide range of practice duration with $38 \%$ (163 of 445) being in practice longer than 20 years.

The most common answers for Scenario 1: spondylolisthesis with mechanical back pain were "laminectomy with posterolateral fusion using pedicle screws" and "PLIF/TLIF," the most common answer for Scenario 2: spondylolisthesis without mechanical back pain were "laminectomy/foraminotomy," "laminectomy with posterolateral fusion using pedicle screws," and "PLIF/TLIF." For the purposes of 
statistical comparisons, the remaining choices were grouped together as "other surgery."

\section{Scenario 1 (spondylolisthesis WITH mechanical back pain)}

Significant differences in treatment choice were observed between surgeons from different regions, and those with different annual operative volume and practice duration (Table 1).

When asked how they would treat the patient with spondylolisthesis and with mechanical back, those in the South East had 2.6 times greater odds of performing PLIF/TLIF as compared to those in the North East who were more likely to perform a laminectomy/foraminotomy ( $\mathrm{p}=0.004)$. Similarly, the surgeons from the North East had 4.8 times greater odds of doing a laminectomy/foraminotomy versus those of the South West, who were more likely to perform "Other" (which includes the various other fusion operations described above).

Comparing surgeons based on annual operative volume, those performing fewer than 100 surgeries per year significantly differed from the rest. Specifically, surgeons performing 101-150 cases/year had 3.9 times greater odds of choosing PLIF/TLIF as compared to those performing 0-100 cases/year who were more likely to choose laminectomy with posterolateral fusion using pedicle screws ( $\mathrm{p}=0.003$ ). Those performing 151-200 had 3.4 times greater odds ( $\mathrm{p}=0.004)$ and those performing 201-250 had 7.6 times greater odds $(\mathrm{p}<0.0001)$.

Surgeons significantly varied based on how long they had been in practice. Surgeons in practice greater than 20 years had 3.9 times greater odds of performing laminectomy with posterolateral fusion/pedicle screws as compared to PLIF/TLIF among those in practice $<5$ years $(\mathrm{p}=0.003)$ and 2.7 times greater odds as compared to PLIF/TLIF among those in practice $5-10$ years $(\mathrm{p}=0.0003)$. 
Scenario 2 (spondylolisthesis WITHOUT mechanical back pain)

Significant differences in treatment choice were observed between surgeons from different specialties (orthopedics versus neurosurgery), and those with different annual operative volume and practice duration (Table 2).

Comparing the responses by specialty, we found that neurosurgeons had 3.4 times greater odds of performing laminectomy/foraminotomy $(\mathrm{p}=0.001)$ and 2.1 times greater odds of performing PLIF/TLIF ( $\mathrm{p}=0.01$ ) as compared to orthopedic surgeons who had greater odds of using a laminectomy with posterolateral fusion and pedicle screws.

Spine surgeons that operated 0-100 cases per year had greater odds of doing laminectomy/foraminotomy as compared to those that operate 151-200 cases per year who were more likely to perform laminectomy with PLF/pedicle screws (OR 3.7, $\mathrm{p}=0.01$ ) or PLIF/TLIF (OR 6.25, $\mathrm{p}=0.003$ ). Those surgeons performing 201-250 cases/year had 2.7 times greater odds of doing PLIF/TLIF as compared to the 0-100 group performing laminectomy with PLF/pedicle screws (OR 4.8, $\mathrm{p}=0.003$ ), laminectomy/foraminotomy ( $\mathrm{OR}=7.7, \mathrm{p}=0.001$ ), or other surgery choices (including ALIF, LIF, interspinous spacer device; OR 4.55, $\mathrm{p}=0.007$ ). Similarly, those performing 251-300 had greater odds of doing PLIF/TLIF versus the 0-100 group who had greater odds of doing laminectomy with PLF/pedicle screws (OR 4.1, $\mathrm{p}=0.008$ ), laminectomy/foraminotomy ( $\mathrm{OR}=5.3, \mathrm{p}=0.006$ ), or "other" (OR 5.3, $p=0.006)$. Lastly, there was a trend towards significance $(p=0.02)$ among the $>300$ group doing PLIF/TLIF as compared to the 0-100 group performing laminectomy with PLF/pedicle screws (OR 3.7, $\mathrm{p}=0.01$ ), laminectomy/foraminotomy (OR 4.8, $\mathrm{p}=0.0009$ ), or "other" (OR 5.0, $\mathrm{p}=0.006$ ).

When comparing surgeons based on how long they had been in practice, those working $>20$ years differed from the other groups. The $>20$ year group had 7.1 times greater odds of choosing laminectomy with PLF/pedicle screws as compared to 
those in practice $<5$ years choosing PLIF/TLIF ( $\mathrm{p}=0.007$ ), 3.5 times greater odds compared to those in practice 5-10 years choosing PLIF/TLIF ( $\mathrm{p}=0.0004$ ), and 2.3 times greater odds as compared to those in practice $10-15$ years $(\mathrm{p}=0.01)$.

\section{Percent Disagreement}

Table 3 demonstrates the level of variability among spine surgeons in selecting a treatment for spondylolisthesis with (S1) and without (S2) associated mechanical back pain. Specifically, we calculated the probability of two randomly chosen surgeons from within specific cohorts (based on region, specialty, etc) disagreeing on treatment choice.

Among all respondents, there was $64 \%$ disagreement for S1 and 71\% disagreement for S2. S2 tended to consistently have a higher probability of disagreement as compared to S1. Only few significant differences in probability of disagreement were identified within each question based on surgeon demographics.

For S1, disagreement differed significantly by practice length, wherein disagreement ranged from a low of 52\% among those practicing 5-10 years to a high of 70\% among those practicing $>20$ years $(\mathrm{OR}=0.47, \mathrm{CI}=0.29,0.73)$. Those in practice 10 15 years also differed significantly less (58\%) in their selection of an operation as

compared to the $70 \%$ among those in practice $>20$ years $(\mathrm{OR}=0.59, \mathrm{CI}=0.35,0.91)$. Disagreement also differed significantly based on specialty, with $56 \%$ probability of disagreement among neurosurgeons as compared to $76 \%$ probability among orthopedic surgeons $(\mathrm{OR}=0.67, \mathrm{CI}=0.44,0.96)$. No other differences were seen in probability of disagreement based on geography, fellowship training, practice volume, or practice type.

For S2 the probability of disagreement was higher than for S1. However, for S2 there were no differences in probability of disagreement based on region, specialty, fellowship training, practice type, practice volume, or duration in practice. 


\section{Discussion}

\section{Overview}

There is substantial clinical equipoise ( $\sim 75$ disagreement) among surgeons on the approach used to treat patients with low back pain. Disagreement is highest in the southwest and lowest in the midwest (82\% vs. 69\%, respectively), and there is significantly lower disagreement among those in academic practices versus those in private/hybrid practices (56\% vs. 79\%, respectively). Those in academic practices have almost 4 times greater odds of choosing no surgery as compared to those in hybrid and private practices, who are more likely to choose ALIF or PLIF/TLIF for the treatment of LBP. Furthermore, those with fellowship training have 2 times greater odds of selecting no surgery and 4 times greater odds of selecting ALIF as compared to those without fellowship training who were more likely to select TLIF/PLIF.

In recent decades spine surgery, as well as many other areas in surgery and medicine, have advanced dramatically. Clinical investigations have led to improved safety and quality, shorter hospital stays, fewer complications, as well as a multitude of novel techniques for a given pathology. Despite these advances, however, there is still substantial optimization needed in terms of identifying the most comparatively effective and most cost effective treatments. As the United States continues into an era of value-based health care, it becomes increasingly important to identify the optimal surgical and medical treatments for patients. Our hypothesis that there would be substantial differences in the approach spine surgeons in the U.S. take in the treatment of back pain was verified by several of the results in this study.

Low back pain (LBP) is one of the most prevalent pathologies, affecting $80 \%$ of the US population at some point in their lives. LBP is the most common pathology that limits activity in people younger than $45,3^{\text {rd }}$ most common cause for surgical procedures, and $5^{\text {th }}$ most common cause for hospital admissions. ${ }^{13}$ Yet despite its prevalence and high financial cost to the health care system, there is still a lack of consensus regarding treatment of patients with LBP. Most agree that nonsurgical 
treatment is the first-line approach. ${ }^{14}$ But among patients that have symptoms refractory to conservative treatment, the decision of whether surgery is appropriate and, if so, what approach should be used is unclear. Accordingly, the purpose of this study was to identify the differences in treatment of LBP, and gain better understanding of the variables that influenced these decisions.

In both clinical scenarios the patient presented had mechanical LBP, with no radicular symptoms, that was refractory to 6-weeks of conservative treatment. The patients also had a discogram that produced concordant symptoms at L4/5 with negative controls at the levels above and below (S1) or a discogram producing symptoms at both L4/5 and L5/S1, with a negative control at the level above (S2). For S1, the most commonly selected treatment options were single level ALIF, single level PLIF/TLIF, and no surgery. For S2, the most common treatment options selected were two level ALIF (with posterior fixation), two level PLIF/TLIF, and no surgery. Many of the significant differences found were similar for both scenarios, but more differences were seen in S2.

\section{Private versus Academic practice}

Surgeons in private practice had three times greater odds of selecting ALIF, PLIF/TLIF, and "other surgery" as compared to "no surgery" which was more seen among the academic cohort (both S1 and S2). It is unclear why this effect was seen and there have been no previous studies that have identified these practice differences. There are several possible explanations. The first is that academic practices tend to be in tertiary referral hospitals that may see fewer cases of mechanical LBP, and rather, the complex cases with LBP that do present tend to have worse outcomes following fusion surgery. Private practice surgeons may also more commonly use discograms relative to their colleagues in academic settings, and therefore rely on those results more heavily. Lastly, another explanation may be related to financial incentives within the private practice versus academic hospital setting (though many academic settings have substantial financial incentives as well). 
We also evaluated the degree of clinical equipoise based on determining probability of disagreement on treatment strategies among randomly selected surgeons. In other words - how likely is it for two surgeons to differ on surgical treatment for a given pathology? For this analysis as well we found that overall there was $76 \%$ and $75 \%$ disagreement for S1 and S2 respectively. But specifically evaluating the impact of practice model, we found that among those in academic practice there was 56\% disagreement, whereas there was 78-79\% disagreement for hybrid and private practice models (OR 3.0, p<0.05). This effect, as well, may be related to the typical patient populations and the heterogeneity seen by surgeons in these various types of practice models.

\section{Specialty and Fellowship training}

For the second scenario, there were additional significant differences between orthopaedic and neurosurgery and based on fellowship training. Orthopaedic surgeons had 3.2 times greater odds of selecting ALIF versus neurosurgeons who were more likely to choose PLIF/TLIF. Additionally, those that were fellowship trained had 4.1 times greater odds choosing ALIF and 2.3 times greater odds choosing no surgery versus those not fellowship trained who were more likely to choose TLIF/PLIF.

The differences in surgical practice patterns based on spine surgical training are not unexpected. There is substantial variability in training that begins during residency, whereby neurosurgery residents log significantly more decompressive surgery cases, but orthopaedic residents perform significantly more spine deformity cases. ${ }^{15}$ Moreover, from the perspective of orthopaedic versus neurosurgery residency program directors, there is a different emphasis placed on the importance of fellowship and whether they feel it is necessary for their respective residents. ${ }^{16}$ Once in practice, Irwin and colleagues ${ }^{17,18}$ found that, among 30 spine surgeons surveyed, orthopaedic surgeons were significantly more likely than neurosurgeons to recommend fusion and instrumentation in certain cases, such as degenerative scoliosis with stenosis. Other survey and database studies ${ }^{19-22}$ have also found differences in surgical decision making between neurosurgeons and orthopaedic 
surgeons. The differences in training, practice cultures, and limited high quality evidence providing clear indications for surgery invariably lead to the differences in practice patterns. In contrast to many of the aforementioned studies, the present investigation both directly queries the surgeons and includes a substantially larger sample size, thereby allowing for superior generalizability and statistical power.

\section{Geographical Location}

Geographical location also affected differences in surgical decision-making. For both $\mathrm{S} 1$ and $\mathrm{S} 2$, surgeons identifying as practicing in the southwest, had $82 \%$ and $85 \%$ disagreement respectively. In contrast, the Midwest had 69\% and $72 \%$ disagreement for S1 and S2 respectively. The northeast and southeast had rates of disagreement that were similar to the Midwest. It is unclear why these differences exist, but there have been several studies that have reported geographical differences in rate of surgery and complications/outcomes. ${ }^{23-28}$ According to the Dartmouth Atlas of Health, ${ }^{29}$ regional variations are dramatic across the country. Using Medicare beneficiary data, they found that spinal decompression was about 8 times more likely in Tacoma, Washington and Mason City, Iowa relative to Bronx, New York (200.7 per 100,000 people versus 25.3 per 100,000 people). For fusions the difference was even more dramatic, with almost a 14-fold higher rate of fusion for Bradenton, Florida relative to Bangor, Maine (127.5/100,000 vs. 9.2/100,000, respectively).

\section{Discogram Use}

The largest difference in percent of disagreement was based on discogram use. Those surgeons that often use discogram had 83\% disagreement, whereas those that never use discogram had $48 \%$ disagreement. This major difference may be related to the controversy that surrounds discogram use for patients with LBP; with some studies highlighting the relatively high false positive rates and possible complications, and others stressing its diagnostic importance. ${ }^{30}$ Based on our data, it is plausible to suggest that those surgeons that commonly use discogram may be more variable in surgical approaches and treatments used for LBP. It is also 
possible that, in general, their treatment paradigm and cost/benefit analysis for patients with LBP is different than their counterparts that never use discograms. This difference is further supported as those using discography often had 25 times greater odds of choosing TLIF/PLIF than those who never performed discograms, who were more likely to choose no surgery.

The differences seen throughout underscore the high variability in practice patterns for LBP based on specialty, training, experience, geographical region, and practice type. The results seen herein parallel the results obtained in our previous paper based on this survey. ${ }^{11}$ The question regarding treatment of two-time recurrent disc herniation also revealed substantial clinical equipoise with relatively high overall disagreement of $69 \%$ (whereas there was only $22 \%$ disagreement for first time lumbar disc herniation). The differences in treatment decisions for disc herniation in the previous study, however, stemmed from differences in duration in practice and annual case volume. ${ }^{11}$ In contrast, the differences seen in the present study looking at treatment of LBP, were based on practice type (private vs academic), specialty, and fellowship training.

The lack of definitive evidence supporting one surgical approach versus another, and the lack of definitive evidence identifying the indications for surgery among this patient population, is a major contributor to this variability. This heterogeneity of delivered care for low back pain, and other spine pathologies, is concerning as it implies patients can present with the same pathology to different surgeons and receive entirely different surgeries, or no surgery. This reality underscores the need for the spine community to investigate in earnest the nonoperative and operative treatments with the most value (quality/cost) to patients. Clearly, generating the appropriately designed and executed comparative effectiveness and cost-effectiveness studies are a necessary step in this process. Another avenue to define value at larger institutions with high surgical volumes and a large number of surgeons is to carefully measure the treatment outcomes and costs across providers for common pathologies. As the U.S. more fully adopts a value-centric delivery of care it is imperative that spine surgeons be proactive in 
defining what works best for their patients, or it is quite possible that such will be dictated to them by other stakeholders (i.e. payors).

There are limitations in the present study that should be recognized. The survey represents only a percentage of spine surgeons currently practicing in the U.S. The case scenarios that were included presented a generic patient with low back pain, no radicular symptoms, that was refractory to conservative therapy; no other information was provided (eg. smoking, psychosocial factors, spondylolisthesis, etc) that would potentially affect the decision-making process. Moreover, the study design and structure of the survey could have had a degree of experimenter bias. However, the cases presented herein represent a very common pathology that is frequently seen by spine surgeons. The wide representation of various spine surgeons from different training and regional backgrounds enables identification of these substantial practice differences.

\section{Conclusion}

This survey study is among the first and the largest to define the variability in surgical decision-making for low back pain based on surgeon characteristics. Recognizing the substantial variability that exists will hopefully spur additional studies aimed at identifying the proper indications as well as the most cost effective treatments for LBP. Patients should be involved in the decision making process to identify the optimal treatment based on their values.

\section{References}

1. Eismont, F. J., Norton, R. P. \& Hirsch, B. P. Surgical management of lumbar degenerative spondylolisthesis. J Am Acad Orthop Surg 22, 203-213 (2014).

2. Weinstein, J. N. et al. Surgical versus nonsurgical treatment for lumbar degenerative spondylolisthesis. N. Engl. J. Med. 356, 2257-2270 (2007).

3. Pearson, A. et al. Predominant leg pain is associated with better surgical outcomes in degenerative spondylolisthesis and spinal stenosis: results from the Spine Patient Outcomes Research Trial (SPORT). Spine 36, 219-229 (2011). 
4. Kristof, R. A. et al. Degenerative lumbar spondylolisthesis-induced radicular compression: nonfusion-related decompression in selected patients without hypermobility on flexion-extension radiographs. J. Neurosurg. 97, 281-286 (2002).

5. Epstein, N. E. Decompression in the surgical management of degenerative spondylolisthesis: advantages of a conservative approach in 290 patients. J Spinal Disord 11, 116-122; discussion 123 (1998).

6. Mulholland, R. C. Degenerative lumbar spondylolisthesis: a meta-analysis of literature 1970-1993. Spine 20, 1957-1958 (1995).

7. Jang, J.-W., Park, J.-H., Hyun, S.-J. \& Rhim, S.-C. Clinical Outcomes and Radiologic Changes Following Microsurgical Bilateral Decompression via a Unilateral Approach in Patients With Lumbar Canal Stenosis and Grade I Degenerative Spondylolisthesis With a Minimum 3-year Follow-up. J Spinal Disord Tech (2012). doi:10.1097/BSD.0b013e31827566a8

8. Herkowitz, H. N. \& Kurz, L. T. Degenerative lumbar spondylolisthesis with spinal stenosis. A prospective study comparing decompression with decompression and intertransverse process arthrodesis. J Bone Joint Surg Am 73, 802-808 (1991).

9. Fischgrund, J. S. et al. 1997 Volvo Award winner in clinical studies. Degenerative lumbar spondylolisthesis with spinal stenosis: a prospective, randomized study comparing decompressive laminectomy and arthrodesis with and without spinal instrumentation. Spine 22, 2807-2812 (1997).

10. Ha, K.-Y., Na, K.-H., Shin, J.-H. \& Kim, K.-W. Comparison of posterolateral fusion with and without additional posterior lumbar interbody fusion for degenerative lumbar spondylolisthesis. J Spinal Disord Tech 21, 229-234 (2008).

11. Mroz, T. E. et al. Differences in the surgical treatment of recurrent lumbar disc herniation among spine surgeons in the United States. Spine J 14, 2334-2343 (2014).

12. Harris, P. A. et al. Research electronic data capture (REDCap)--A metadatadriven methodology and workflow process for providing translational research informatics support. Journal of Biomedical Informatics 42, 377-381 (2009).

13. Andersson, G. B. Epidemiological features of chronic low-back pain. Lancet 354, 581-585 (1999).

14. Fritzell, P., Hägg, O., Wessberg, P. \& Nordwall, A. 2001 Volvo Award Winner in Clinical Studies: Lumbar fusion versus nonsurgical treatment for chronic low back pain: a multicenter randomized controlled trial from the Swedish Lumbar Spine Study Group. Spine 26, 2521-2532; discussion 2532-2534 (2001).

15. Daniels, A. H., Ames, C. P., Smith, J. S. \& Hart, R. A. Variability in spine surgery procedures performed during orthopaedic and neurological surgery residency training: an analysis of ACGME case log data. J Bone Joint Surg Am 96, e196 (2014).

16. Daniels, A. H. et al. The Current State of United States Spine Surgery Training: A Survey of Residency and Spine Fellowship Program Directors. Spine Deformity 2, 176-185 (2014).

17. Irwin, Z. N. et al. Variation in surgical decision making for degenerative spinal disorders. Part I: lumbar spine. Spine 30, 2208-2213 (2005). 
18. Irwin, Z. N. et al. Variation in surgical decision making for degenerative spinal disorders. Part II: cervical spine. Spine 30, 2214-2219 (2005).

19. Hussain, M., Nasir, S., Moed, A. \& Murtaza, G. Variations in Practice Patterns among Neurosurgeons and Orthopaedic Surgeons in the Management of Spinal Disorders. Asian Spine J 5, 208-212 (2011).

20. Arnold, P. M. et al. Differences between neurosurgeons and orthopedic surgeons in classifying cervical dislocation injuries and making assessment and treatment decisions: a multicenter reliability study. Am J. Orthop. 38, E156-161 (2009).

21. Raja Rampersaud, Y. et al. Agreement between orthopedic surgeons and neurosurgeons regarding a new algorithm for the treatment of thoracolumbar injuries: a multicenter reliability study. J Spinal Disord Tech 19, 477-482 (2006).

22. Bederman, S. S. et al. The who, what and when of surgery for the degenerative lumbar spine: a population-based study of surgeon factors, surgical procedures, recent trends and reoperation rates. Can J Surg 52, 283-290 (2009).

23. Weinstein, J. N., Lurie, J. D., Olson, P. R., Bronner, K. K. \& Fisher, E. S. United States' trends and regional variations in lumbar spine surgery: 1992-2003. Spine 31, 2707-2714 (2006).

24. Cook, C. et al. Geographic variation in lumbar fusion for degenerative disorders: 1990 to 2000. Spine J 7, 552-557 (2007).

25. Nilasena, D. S., Vaughn, R. J., Mori, M. \& Lyon, J. L. Surgical trends in the treatment of diseases of the lumbar spine in Utah's Medicare population, 1984 to 1990. Med Care 33, 585-597 (1995).

26. Ciol, M. A., Deyo, R. A., Howell, E. \& Kreif, S. An assessment of surgery for spinal stenosis: time trends, geographic variations, complications, and reoperations. J Am Geriatr Soc 44, 285-290 (1996).

27. Cherkin, D. C., Deyo, R. A., Loeser, J. D., Bush, T. \& Waddell, G. An international comparison of back surgery rates. Spine 19, 1201-1206 (1994).

28. Deyo, R. A. \& Mirza, S. K. Trends and variations in the use of spine surgery. Clin. Orthop. Relat. Res. 443, 139-146 (2006).

29. Fisher, E. S. et al. Variation in the Care of Surgical Conditions: Spinal Stenosis.

30. Walker, J., El Abd, O., Isaac, Z. \& Muzin, S. Discography in practice: a clinical and historical review. Curr Rev Musculoskelet Med 1, 69-83 (2008). 